Corrigendum

\title{
Corrigendum to "Effects of Number of Repetitions and Number of Hours of Shaping Practice during Constraint-Induced Movement Therapy: A Randomized Controlled Trial"
}

\begin{abstract}
Auwal Abdullahi
Department of Physiotherapy, Bayero University Kano, Kano, Kano State, Nigeria

Correspondence should be addressed to Auwal Abdullahi; aabdullahi.pth@buk.edu.ng

Received 28 July 2020; Accepted 28 July 2020; Published 24 August 2020

Copyright (c) 2020 Auwal Abdullahi. This is an open access article distributed under the Creative Commons Attribution License, which permits unrestricted use, distribution, and reproduction in any medium, provided the original work is properly cited.
\end{abstract}

In the article titled "Effects of Number of Repetitions and Number of Hours of Shaping Practice during ConstraintInduced Movement Therapy: A Randomized Controlled Trial" [1], there was an error in Table 1 in which the incorrect data were given for sex, type of stroke, hand dominance, and side affected. The author apologises for this error and the corrected table is shown below.

TABLE 1: Baseline characteristics of the study participants.

\begin{tabular}{|c|c|c|c|c|c|c|}
\hline \multirow{2}{*}{ Variable } & \multirow{2}{*}{ Control $(n=12)$} & \multicolumn{2}{|c|}{ Group } & \multirow[b]{2}{*}{$600 \operatorname{Rep}(n=11)$} & \multirow{2}{*}{ Statistics } & \multirow{2}{*}{$p$} \\
\hline & & Modified CIMT $(n=13)$ & $300 \operatorname{Rep}(n=12)$ & & & \\
\hline Age & $58.83 \pm 10.57$ & $54.62 \pm 6.00$ & $59.42 \pm 13.93$ & $57.60 \pm 10.27$ & & \\
\hline Time since stroke & $19.89 \pm 7.20$ & $14.75 \pm 4.46$ & $21.67 \pm 6.38$ & $13.50 \pm 7.39$ & & \\
\hline $\operatorname{Sex}(M / F)$ & $9 / 3$ & $9 / 4$ & $4 / 8$ & $5 / 6$ & & \\
\hline Type of stroke $(\mathrm{I} / \mathrm{H})$ & $8 / 4$ & $10 / 3$ & $11 / 1$ & $7 / 4$ & & \\
\hline Hand dominance $(\mathrm{R} / \mathrm{L})$ & $12 / 0$ & $12 / 1$ & $11 / 1$ & $10 / 1$ & & \\
\hline Side affected $(\mathrm{R} / \mathrm{L})$ & $7 / 5$ & $9 / 4$ & $7 / 5$ & $8 / 3$ & & \\
\hline MAL (how well) & $2.13 \pm 1.32$ & $1.62 \pm 0.73$ & $2.30 \pm 1.16$ & $1.51 \pm 0.94$ & $F=1.577$ & 0.208 \\
\hline MAL (amount of use) & $1.91 \pm 1.37$ & $1.75 \pm 0.54$ & $2.31 \pm 1.19$ & $1.18 \pm 0.77$ & $F=2.433$ & 0.078 \\
\hline WMFT & $2.22 \pm 1.19$ & $2.17 \pm 0.82$ & $1.05 \pm 0.30$ & $1.99 \pm 0.52$ & $F=0.524$ & 0.668 \\
\hline FM & $31.42 \pm 16.21$ & $34.00 \pm 14.15$ & $35.17 \pm 13.02$ & $30.45 \pm 10.76$ & $F=0.299$ & 0.826 \\
\hline UPSET & $4.75 \pm 3.26$ & $4.58 \pm 2.21$ & $4.74 \pm 1.88$ & $4.56 \pm 1.81$ & $F=0.021$ & 0.996 \\
\hline
\end{tabular}

$\mathrm{R} / \mathrm{L}=$ right/left; $\mathrm{I} / \mathrm{H}=$ ischaemic/haemorrhagic; $\mathrm{M} / \mathrm{F}=$ male/female. 


\section{References}

[1] A. Abdullahi, "Effects of number of repetitions and number of hours of shaping practice during constraint-induced movement therapy: a randomized controlled trial," Neurology Research International, vol. 2018, Article ID 5496408, 9 pages, 2018. 